\title{
Luminescence Hole Burning and Quantum Size Effect of Charged Excitons in $\mathrm{CuCl}$ Quantum Dots
}

\author{
Tadashi Kawazoe and Yasuaki Masumoto \\ Institute of Physics and Center for TARA (Tsukuba Advanced Research Alliance), University of Tsukuba, \\ Tsukuba, Ibaraki 305, Japan
}

(Received 12 August 1996)

\begin{abstract}
Luminescence hole-burning phenomena were observed in $\mathrm{CuCl}$ quantum dots embedded in a $\mathrm{NaCl}$ crystal. As a result of the selective excitation of the $Z_{3}$ exciton band of $\mathrm{CuCl}$ quantum dots, a resonantly burned hole and moreover its sidebands appear in the luminescence spectrum. The Stokes shift of the sidebands increases with the increase in the burning photon energy, and its dependence is explained by the quantum size effect of the negatively charged exciton $X^{-}$and the positively charged exciton $X_{2}{ }^{+}$. New exciton complexes, $X^{-}$and $X_{2}{ }^{+}$, confined in quantum dots were first observed by the luminescence hole burning. [S0031-9007(96)01828-5]
\end{abstract}

PACS numbers: 73.20.Dx, 71.35.-y, 78.40.Ha, 78.55.Fv

The quantum confinement effect for electrons, holes, and excitons in semiconductor quantum dots (QDs) has been studied extensively [1]. Many experimental results are systematically explained by a ratio of QD radius to the exciton Bohr radius [2]. In this sense, understanding of the quantum size effect for electrons, holes, and excitons is in the matured stage. However, the effect for bound exciton complexes in semiconductor QDs have not been investigated so far. It was pointed out that exciton complexes in semiconductor QDs play an important role in interesting and applicable phenomena, such as persistent spectral hole burning (PSHB) [3-6]. Therefore, the study of exciton complexes in semiconductor QDs is valuable to clarify their quantum size effect and the mechanism of the PSHB.

Excitons are bound to impurities or structural defects. In a bulk $\mathrm{CuCl}$ crystal, bound excitons are observed as the $I_{1}$ and $I_{2}$ luminescence lines [7]. In addition, the existence of positively $\left(X_{2}^{+}\right)$and negatively $\left(X^{-}\right)$charged excitons were predicted by Lampert [8], and its existence in $\mathrm{CuCl}$ was suggested by Stébé et al. [9]. A negatively charged exciton is a complex composed of two electrons and a hole, analogous to $H^{-}$. A positively charged exciton is a complex composed of an electron and two holes, analogous to $H_{2}^{+}$. They have been experimentally confirmed in CdTe and GaAs quantum wells recently [10,11]. Stébé and Ainane pointed out that the binding energy of the charged exciton is enhanced in a quantum well [12]. However, the charged exciton has never been observed in QDs. In this paper, we report the observation of the charged exciton in $\mathrm{CuCl}$ QDs and discuss their quantum size effect. The study is initiated by the observation of the luminescence hole burning (LHB) in $\mathrm{CuCl}$ QDs.

The formation of a spectral hole in luminescence, socalled luminescence hole burning, was first reported in a dye by Gorokhovski et al. in 1974 and 1976 [13,14]. In spite of a long history of the phenomenon, it has been studied compared with the absorption hole burning. The LHB can give us additional information which the absorption hole burning cannot. An absorption spectrum simply reflects the joint density of initial and final states of the transition. On the other hand, a luminescence spectrum reflects the relaxation mode of the luminescent excited states as well as the joint density of states. Therefore, in the LHB, the lowest-energy luminescent excited states, such as bound excitons and the charged excitons, are sensitively observed. The absorption hole spectrum corresponds to the absorption spectrum of the QDs excited selectively. In the same way, the luminescence hole spectrum is recognized as the luminescence spectrum of the QDs excited selectively. The fluorescence line narrowing (FLN) affords us similar information as the LHB does. However, it is sometimes difficult to obtain the spectrum near the excitation photon energy because of the scattering of the excitation light. In the LHB experiment, on the other hand, it is comparatively easy to observe the accurate hole spectrum even just at the burning energy, as is mentioned later. Moreover, in case of the semiconductor QDs, the FLN signal is reduced, because the absorption of the selectively excited QDs is reduced with very large burning efficiency [15]. In these senses, the LHB experiment is superior to the FLN experiment.

First, the peculiar characteristics of the luminescence in $\mathrm{CuCl}$ QDs are explained in connection with the LHB. Figure 1 shows absorption, luminescence, and absorption change spectra of the $Z_{3}$ exciton band in $\mathrm{CuCl}$ QDs embedded in a $\mathrm{NaCl}$ crystal at $2 \mathrm{~K}$. The luminescence was measured under the band-to-band excitation at $3.49 \mathrm{eV}$. The absorption spectra and the luminescence spectra inhomogeneously broadened partly because of the size distribution of QDs. The oscillatory fine structures come from a stepwise growth of the $\mathrm{CuCl}$ quantum cubes in a $\mathrm{NaCl}$ crystal [16]. Below $30 \mathrm{~K}$, the luminescence peak shows the large Stokes shift in $\mathrm{CuCl}$ QDs in a $\mathrm{NaCl}$ crystal. It may be explained by the recombination of bound excitons [17]. However, an attentive investigation of the luminescence was necessary for the definitive identification of the luminescence origin, because the luminescence spectrum changes with the increase of the accumulated light ex- 


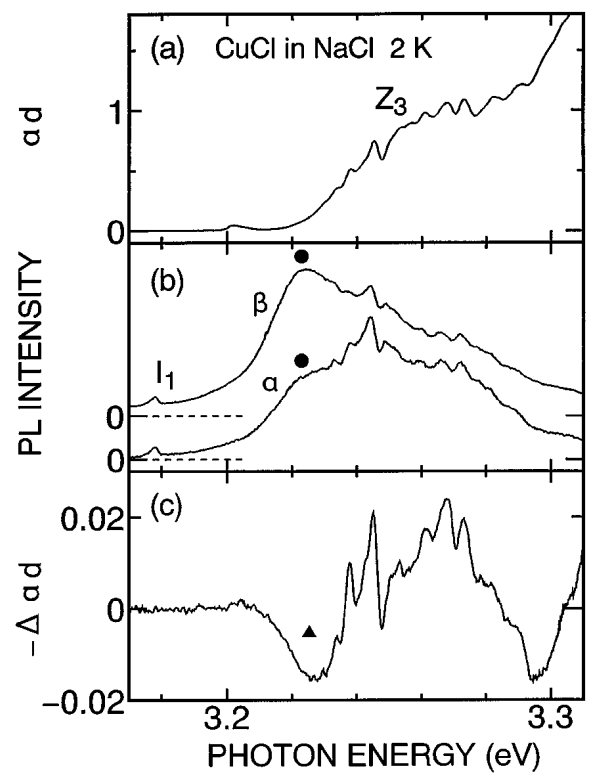

FIG. 1. (a) $Z_{3}$-exciton absorption, (b) luminescence, and (c) absorption change spectra of $\mathrm{CuCl}$ quantum dots in a $\mathrm{NaCl}$ crystal at $2 \mathrm{~K}$.

posure. The luminescence spectral change is shown in Fig. 1(b). The luminescence signals that were accumulated during the sample was excited by 9000 shots of $3.49 \mathrm{eV}$ pulses with the energy density of $0.1 \mu \mathrm{J} / \mathrm{cm}^{2}$. The spectra $\alpha$ and $\beta$ correspond to luminescence after the sample was exposed to accumulated energy density of 0 and $9.9 \mathrm{~mJ} / \mathrm{cm}^{2}$ of the $3.49 \mathrm{eV}$ pulses, respectively. At low accumulated energy density, the luminescence peak almost agrees with the absorption peak. Therefore, the luminescence spectrum $\alpha$ is considered to come from the recombination of free excitons confined in the dots. With the increase of the accumulated energy density, the luminescence at the lower-energy tail marked by a solid circle grows. It is quenched after the thermal annealing cycle. The phenomena are similar to thermally annealing hole filling [3-6], and the thermal quenching of the luminescence elongation caused by the light exposure [6].

Figure 1(c) shows the absorption spectral change after the luminescence changes from $\alpha$ to $\beta$. The minute structures in the differential absorption spectrum corresponds to those in the absorption spectrum one by one, and the induced absorption marked by $\boldsymbol{\Delta}$ appears. The absorption change takes place together with the luminescence change. The energy position of the induced absorption band $\boldsymbol{\Delta}$ corresponds to that of the induced luminescence band $\boldsymbol{Q}$. Both the spectral changes are persistent. We consider that the induced luminescence band comes from the charged exciton and is formed by the photoionization of QDs.

A scenario for the photoionization of QDs is the same as described for the mechanism of the PSHB phenomena [4-6]. First, an exciton is created by the light absorption. Second, the exciton is localized at the surface of the QD. Next, the exciton is dissociated and an electron or a hole tunnels through the potential barrier in the host and is trapped at a bound center in the host. Another carrier remains in the QD, and the dot is ionized. According to previous works [10,11], a prerequisite for the observation of a negatively charged exciton is that sufficiently high density of electrons should exist for the observation and that the electron density must not be so high as to screen the Coulomb interaction working inside the exciton. For example, in $n$-doped $\left(\sim 10^{16} \mathrm{~cm}^{-3}\right)$ GaAs quantum wells and CdTe quantum wells, the negatively charged excitons were observed together with excitons. When the charged exciton is observed, the exciton structure decreases and the charged exciton structure increases in the optical spectrum with increasing the electron density. At higher electron density, exciton and charged exciton structures decrease and are replaced by broad, structureless absorption and emission peaks showing a Fermi-edge singularity. Supposing that an electron is trapped in a QD whose radius is $5 \mathrm{~nm}$, the electron density becomes $2 \times 10^{18} \mathrm{~cm}^{-3}$. The electron density is much higher than that in the previous reports of the charged excitons in $\mathrm{GaAs}$ and CdTe. In a $\mathrm{CuCl}$ crystal, an exciton Bohr radius is much smaller than the radius in GaAs or CdTe crystals. Therefore, the Coulomb screening hardly takes place in $\mathrm{CuCl}$. We consider that the density is not unreasonable for the observation of the charged exciton in a $\mathrm{CuCl}$ crystal.

The LHB experiment gives us detailed information for the induced luminescence band. The experimental result definitely shows that the induced luminescence band comes from the recombination of charged excitons confined in QDs. The laser system used in the LHB experiment was a $Q$-switched $\mathrm{Nd}^{3+}$ : YAG laser and a dye laser pumped by it. The excitation photon energy of the dye laser was tuned to the $Z_{3}$ exciton band of $\mathrm{CuCl}$ QDs. The spectral linewidth of the dye laser was $0.014 \mathrm{meV}$, which was much narrower than the instrumental resolution of the experiment. The third harmonics of the $\mathrm{Nd}^{3+}$ : YAG laser $(3.49 \mathrm{eV})$ was used as a band-to-band excitation light source for the measurement of the luminescence at $2 \mathrm{~K}$. The laser pulse width was $5 \mathrm{~ns}$, and the repetition rate was $30 \mathrm{~Hz}$. For the observation of the LHB, the sample was excited alternatively by $3.49 \mathrm{eV}$ and dye laser pulses, and a synchronized chopper selectively collects luminescence generated by the $3.49 \mathrm{eV}$ excitation. When the dye laser pulse excited the sample, the chopper shut the path between the sample and the monochromator. At $33 \mathrm{~ms}$ after the dye laser pumped the sample, the $3.49 \mathrm{eV}$ pulse excited the sample for the measurement of luminescence and the chopper opened the path. The luminescence lifetime of the sample, measured experimentally, was $4 \mathrm{~ns}$ at $2 \mathrm{~K}$. The alternative excitation method is suitable for the observation of luminescence of the sample exposed to the dye laser, because the $3.49 \mathrm{eV}$ excitation causes the hole filling. The luminescence produced by the $3.49 \mathrm{eV}$ excitation is observed in this way.

Figure 2(a) shows the absorption spectrum of $\mathrm{CuCl}$ QDs embedded in a $\mathrm{NaCl}$ host crystal at $2 \mathrm{~K}$. The $Z_{3}$ exciton 
absorption peak shows a blueshift of $20 \mathrm{meV}$ from its energy of a $\mathrm{CuCl}$ bulk crystal. The blueshift is explained by the exciton confinement model [1,2]. An average radius of the $\mathrm{CuCl}$ QDs estimated on the exciton confinement model is $3.0 \mathrm{~nm}$. Figure 2(b) shows the luminescence spectra of the sample. The sample was exposed to the preexcitation of the $3.49 \mathrm{eV}$ pulses with the accumulated fluence density of $10 \mathrm{~mJ} / \mathrm{cm}^{2}$. The luminescence signals were accumulated while the sample was excited by 900 shots of the $3.49 \mathrm{eV}$ pulses with the energy density of $0.5 \mu \mathrm{J} / \mathrm{cm}^{2}$. The luminescence change caused by the $3.49 \mathrm{eV}$ pulses for the luminescence observation was avoided by the preexcitation, because the luminescence change comes to an end after the excitation by the $3.49 \mathrm{eV}$ pulses reaches the accumulated energy density of $10 \mathrm{~mJ} / \mathrm{cm}^{2}$. A dashed line shows the luminescence spectrum before the sample is exposed to the dye laser pulses. The luminescence peak is located at $3.206 \mathrm{eV}$, which is lower than the absorption peak of the $Z_{3}$ exciton, $3.223 \mathrm{eV}$. This Stokes shift of the luminescence was caused by the light preexposure. A solid line shows the luminescence spectrum, after the $3.220 \mathrm{eV}$ narrow-band dye laser pulses exposed the sample with the energy density of $10 \mu \mathrm{J} / \mathrm{cm}^{2}$. The burning energy position is indicated by an arrow in Fig. 2(a). The luminescence shows a sharp hole $(X)$ at the burning energy in the spectrum. Much larger satellite holes $A, B$, and $\bullet$ are observed at the lower-energy side of the burning laser energy. At the high energy side, the luminescence intensity increases. Figure 2(c) shows the absorption spectral change $-\Delta \alpha d$ after the LHB experiment. The luminescence-hole

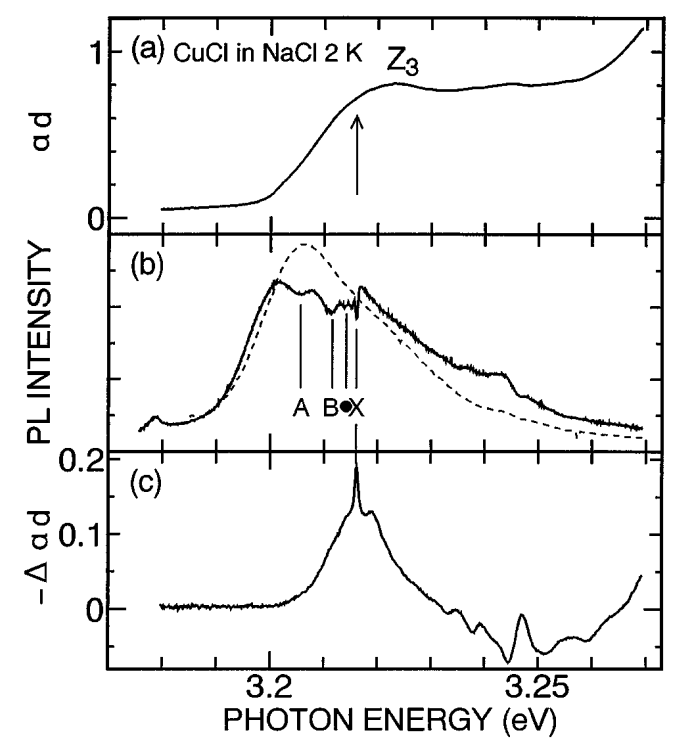

FIG. 2. (a) Absorption spectrum of $\mathrm{CuCl}$ quantum dots embedded in a $\mathrm{NaCl}$ crystal at $2 \mathrm{~K}$. An arrow shows the burning energy of the dye laser. (b) Luminescence spectra before and after the sample is exposed at $3.220 \mathrm{eV}$ with the excitation density of $10 \mu \mathrm{J} / \mathrm{cm}^{2}$. Luminescence spectra was taken under the excitation at $3.49 \mathrm{eV}$ with the excitation density of $0.5 \mu \mathrm{J} / \mathrm{cm}^{2}$. (c) Absorption spectral change of the sample exposed to the narrow-band dye laser at $3.220 \mathrm{eV}$. spectrum gives us information which the absorption-hole spectrum does not.

Figure 3 shows the luminescence-decrease spectra. The luminescence-decrease spectrum is defined by the luminescence spectrum of the sample exposed to the dye laser pulses minus that of the sample which is not exposed to the dye laser pulses. Arrows in the figure indicate the burning energies. Every spectrum has a sharp hole $X$ at the burning energy, and much larger satellite holes, $A, A^{\prime}, B$, and -. With the increase in the burning energy, satellite hole structures are shifted toward the higher-energy side, and their separation increases. As the burning photon energy increases, the depth of the sharp resonant hole becomes smaller and a new structure $\left(A^{\prime}\right)$ grows up. The luminescence hole reflects the luminescence of selectively burned QDs. The peak positions of the structures $A, B$, and $A^{\prime}$ depend on the excitation photon energy.

Figure 4 shows the Stokes shift of the luminescencehole structures as a function of the burning photon energy. Here the Stokes shift means the energy differences between the burning laser and the luminescence-hole structures. The peak $X$ comes from the luminescence of the resonantly excited $Z_{3}$ free exciton confined in QDs, because the energy position of the peak $(X)$ coincides with the burning energy. The solid lines labeled by $C$ and $D$ correspond to a confined transverse acoustic phonon energy and a confined longitudinal acoustic phonon energy, respectively [18]. They explain the Stokes shift of the peaks labeled by - in Fig. 3, whose energy is lower than the burning energy by a few meV. Therefore, the luminescence-decrease peaks $\bullet$ are ascribed to the luminescence of the $Z_{3}$ exciton accompanied by the emission of acoustic phonons. The peaks in the luminescencedecrease spectrum, $A^{\prime}, A$, and $B$, show large Stokes shifts, and depend strongly on the burning photon energy. The positions of the $A^{\prime}$ structure seem to approach the energy position of $I_{1}$ bound exciton in bulk $\mathrm{CuCl}$, as the burn-

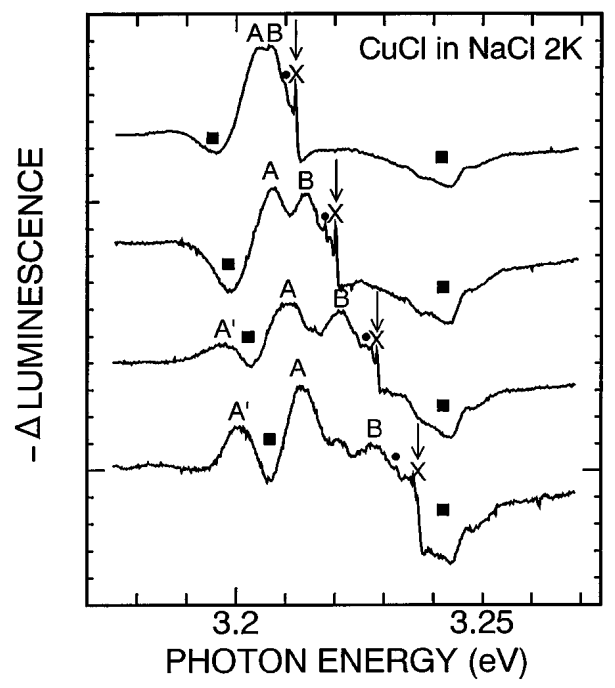

FIG. 3. Photoluminescence decrease at $2 \mathrm{~K}$. Arrows indicate the burning energy. 


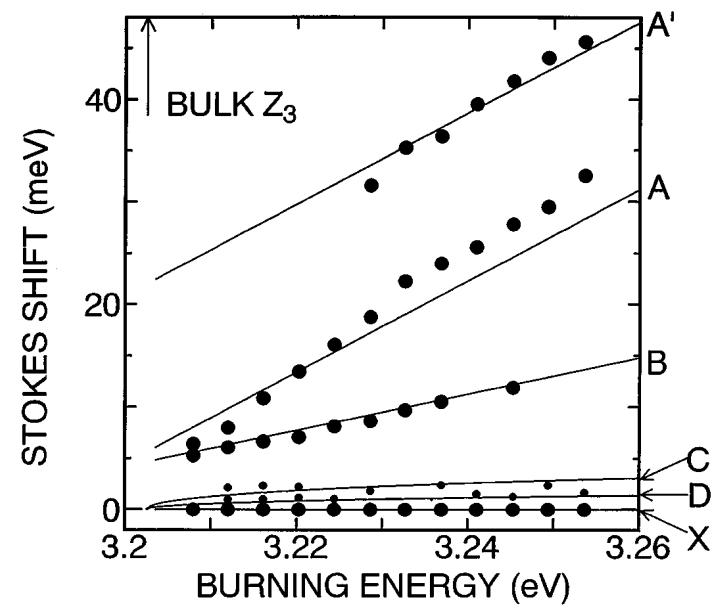

FIG. 4. The burning-energy dependence of the luminescence hole spectra. The burning energy is the photon energy of the dye laser. The Stokes shift is the energy difference between the burning laser and the hole structures.

ing energy approaches the $Z_{3}$-exciton energy in the bulk crystal [7]. Therefore, $\left(A^{\prime}\right)$ structure is assigned to the $I_{1}$ bound exciton. On the other hand, structures, $A$ and $B$, are ascribed to the charged exciton luminescence as is verified in the following.

We consider the quantum confinement of the translational motion of the charged exciton. The translational masses of negatively and positively charged excitons, $M^{-}$and $M_{2}^{+}$, are given by $M^{-}=2 m_{e}^{*}+m_{h}^{*}$ and $M_{2}^{+}=m_{e}^{*}+2 m_{h}^{*}$, where $m_{e}^{*}=0.5 m_{0}$ and $m_{h}^{*}=1.8 m_{0}$ are effective masses of an electron and a hole in a $\mathrm{CuCl}$ crystal, respectively [7]. The energy shifts due to the quantum confinement effect are represented by $\Delta E^{-}=\hbar^{2} \pi^{2} / 2 M^{-} R^{2}$, for the negatively charged exciton, and $\Delta E^{+}=\hbar^{2} \pi^{2} / 2 M_{2}^{+} R^{2}$, for the positively charged exciton, where $R$ is the effective radius of the QD defined by radius of the QD minus half of the exciton Bohr radius [19]. Then, the energy separations, $-\Delta E_{s}^{-}$ and $-\Delta E_{s}^{+}$, between the quantum confined free exciton and the quantum confined charged exciton is given by

$$
\begin{aligned}
-\Delta E_{s}^{-} & =\hbar^{2} \pi^{2} / 2 M R^{2}-\hbar^{2} \pi^{2} / 2 M^{-} R^{2}+E_{\text {bind }}^{-} \\
& =0.18 \times\left(E_{\text {free }}-3.2025\right)+E_{\text {bind }}^{-}(e V),
\end{aligned}
$$

and

$$
\begin{aligned}
-\Delta E_{s}^{-} & =\hbar^{2} \pi^{2} / 2 M R^{2}-\hbar^{2} \pi^{2} / 2 M_{2}^{+} R^{2}+E_{\text {bind }}^{+} \\
& =0.44 \times\left(E_{\text {free }}-3.2025\right)+E_{\text {bind }}^{+}(\mathrm{eV}),
\end{aligned}
$$

where $E_{\text {bind }}^{-}$and $E_{\text {bind }}^{+}$are the binding energy of the charged exciton in bulk $\mathrm{CuCl}$, and $E_{\text {free }}$ is the energy of the free exciton confined in QDs given by $E_{\text {free }}=$ $E_{\text {bulk }}+\hbar^{2} \pi^{2} / 2 M R^{2}$, where $E_{\text {bulk }}$ and $M$ are the exciton resonant energy and the exciton translational mass of a bulk $\mathrm{CuCl}$ [1]. In Eqs. (1) and (2), the value of $3.2025 \mathrm{eV}$ is the $Z_{3}$ free exciton energy in bulk $\mathrm{CuCl}$ [7].

Solid lines $A$ and $B$ in Fig. 4 show the calculated results of (1) and (2) with $E_{\text {bind }}^{-}=4.7 \mathrm{meV}$ and $E_{\text {bind }}^{+}=$
$5.6 \mathrm{meV}$, respectively. The binding energy of $X^{-}$and $X_{2}^{+}$ in bulk $\mathrm{CuCl}$ were set to be 4.7 and $5.6 \mathrm{meV}$, respectively, based on the calculation by Stébé et al. [9]. The solid lines $A$ and $B$ agree with the experimental points. Therefore, we consider the structures $A$ and $B$, in Figs. 2 and 3, come from the recombination of the positively charged exciton and negatively charged exciton, respectively.

As is seen in Fig. 4, the burning energy dependence of the $A^{\prime}$ series coming from the $I_{1}$ bound exciton structure, resembles that of $A$ series. The $I_{1}$ line denoted also by $A^{0} X$ comes from the recombination of the exciton bound to a neutral acceptor. Therefore, $A^{0} X$ can be regarded as an excitonic molecule whose constituent electron is pinned and can be considered as the complex $X_{2}^{+}$going around the pinned electron. In this sense, the $I_{1}$ bound exciton resembles the $X_{2}^{+}$charged exciton. Similarly to the positively charged exciton $X_{2}^{+}$, the translational motion of the $I_{1}$ bound exciton is confined in the QDs. Therefore, the burning energy dependence of the $A^{\prime}$ series resembles that of the $A$ series. The solid line $A$ is calculated by Eq. (2), where $E_{\text {bind }}^{+}=22 \mathrm{meV}$ is the binding energy of the $I_{1}$ bound exciton [7].

In summary, the luminescence spectral change induced by the accumulated light exposure and the LHB phenomena were observed in $\mathrm{CuCl}$ QDs in $\mathrm{NaCl}$ crystal. The luminescence spectral change was understood by the photoionization of the QDs and the creation of the charged exciton states. This conjecture was verified by the study of the LHB. The Stokes shift of the luminescence sideband holes can be explained by the quantum confinement of the charged excitons. This is the first observation of the charged excitons in QDs.

[1] A. I. Ekimov et al., Solid State Commun. 56, 921 (1985); see also A. D. Yoffe, Adv. Phys. 42, 173 (1993).

[2] Y. Kayanuma, Phys. Rev. B 38, 9797 (1988).

[3] K. Naoe et al., Phys. Rev. B 50, 18200 (1994).

[4] Y. Masumoto et al., Phys. Status Solidi B 188, 209 (1995).

[5] Y. Masumoto et al., Phys. Rev. B 52, 4688 (1995).

[6] Y. Masumoto et al., Phys. Rev. B 52, 7834 (1995).

[7] M. Ueta et al., Excitonic Processes in Solids (Springer, Berlin, 1989).

[8] M. A. Lampert, Phys. Rev. Lett. 1, 450 (1958).

[9] B. Stébé and C. Comte, Phys. Rev. B 15, 3967 (1977).

[10] K. Kheng et al., Phys. Rev. Lett. 71, 1752 (1993).

[11] G. Finkelstein et al., Phys. Rev. Lett. 74, 976 (1995).

[12] S. Stébé and A. Ainane, Superlattices Microstruct. 5, 545 (1989).

[13] A. A. Gorokhovski et al., JETP Lett. 20, 216 (1974).

[14] A. A. Gorokhovski and L. A. Rebane, Opt. Commun. 20, 144 (1976).

[15] The quantum efficiency of the hole burning for $\mathrm{CuCl}$ quantum dots in $\mathrm{NaCl}$ was found to be 0.015 at $2 \mathrm{~K}$.

[16] T. Itoh et al., J. Lumin. 60\&61, 396 (1994).

[17] S. Yano et al., Jpn. J. Appl. Phys. 34, 140 (1994).

[18] S. Okamoto and Y. Masumoto, J. Lumin. 64, 253 (1995).

[19] T. Itoh et al., Phys. Status Solidi B 145, 567 (1988). 